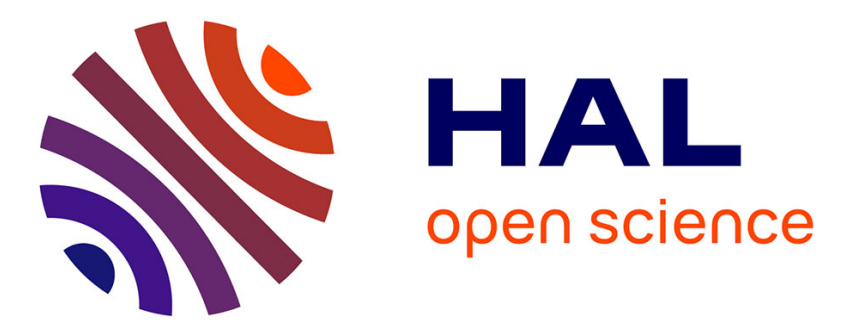

\title{
Dopamine transporter imaging for the diagnosis of multiple system atrophy cerebellar type
}

S. Vergnet, F. Hives, Alexandra Foubert-Samier, P. Payoux, Philippe

Fernandez, Marie Meyer, J. Dupouy, C. Brefel-Courbon, F. Ory-Magne, O. Rascol, et al.

\section{To cite this version:}

S. Vergnet, F. Hives, Alexandra Foubert-Samier, P. Payoux, Philippe Fernandez, et al.. Dopamine transporter imaging for the diagnosis of multiple system atrophy cerebellar type. Parkinsonism \& Related Disorders, 2019, 63, pp.199-203. 10.1016/j.parkreldis.2019.02.006 . hal-03163693

\section{HAL Id: hal-03163693 https://hal.science/hal-03163693}

Submitted on 25 Oct 2021

HAL is a multi-disciplinary open access archive for the deposit and dissemination of scientific research documents, whether they are published or not. The documents may come from teaching and research institutions in France or abroad, or from public or private research centers.
L'archive ouverte pluridisciplinaire $\mathbf{H A L}$, est destinée au dépôt et à la diffusion de documents scientifiques de niveau recherche, publiés ou non, émanant des établissements d'enseignement et de recherche français ou étrangers, des laboratoires publics ou privés.

\section{다)(1) $(5$}

Distributed under a Creative Commons Attribution - NonCommerciall 4.0 International 


\section{Dopamine transporter imaging for the diagnosis of multiple system atrophy cerebellar type}

Sylvain Vergnet, $\mathrm{MD}^{\mathrm{a}}$, Florent Hives, $\mathrm{MD}^{\mathrm{b}}$, Alexandra Foubert-Samier, $\mathrm{MD}, \mathrm{PhD}^{\mathrm{a}, \mathrm{c}}$,

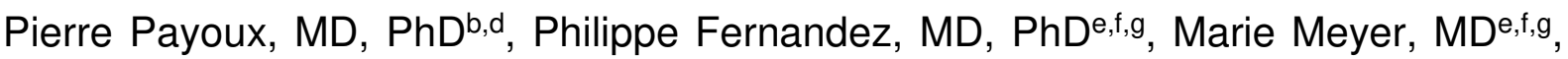
Julia Dupouy, $\mathrm{MD}^{\mathrm{h}}$, Christine Brefel-Courbon, $\mathrm{MD}, \mathrm{PhD}^{\mathrm{h}}$, Fabienne Ory-Magne, $\mathrm{MD}^{\mathrm{h}}$, Olivier Rascol, MD, PhDi,j, François Tison, MD, PhD ${ }^{a, k, l}$, Anne Pavy-Le Traon, MD, PhDj, Wassilios G. Meissner, MD, PhDa,k,l,m

aService de Neurologie, CRMR Atrophie Multisystématisée, CHU Bordeaux, 33000 Bordeaux, France

bService de Médecine Nucléaire, CHU Toulouse Purpan, 31059 Toulouse cedex, France

'INSERM U 897, Public Health and Development Institute (ISPED), Bordeaux University, Bordeaux, France

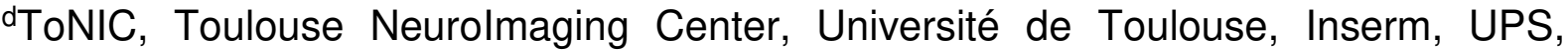
France

eService de Médecine Nucléaire, Pellegrin, CHU de Bordeaux, Bordeaux, France 'INCIA, UMR CNRS 5287, 33000 Bordeaux, France

gUniv. de Bordeaux, 33000 Bordeaux, France

hService de Neurologie et de Pharmacologie, CHU de Toulouse, INSERM U1214, Toulouse University, Toulouse, France

'Université de Toulouse 3, CHU de Toulouse, INSERM, Centre de reference AMS, Service de Neurologie et de Pharmacologie Clinique, Centre d'Investigation Clinique 
CIC1436, Réseau NS-Park/FCRIN et Centre of excellence for neurodegenerative disorders (COEN) de Toulouse, Toulouse, France

iService de Neurologie, CRMR Atrophie Multisystématisée, CHU Toulouse 31059 Toulouse Cedex 9 - INSERM U 1048 Institut des Maladies Métaboliques et Cardiovasculaires, 31432 Toulouse Cedex 4, France

kUniv. de Bordeaux, Institut des Maladies Neurodégénératives, UMR 5293, 33000 Bordeaux, France

'CNRS, Institut des Maladies Neurodégénératives, UMR 5293, 33000 Bordeaux, France

mDept. Medicine, University of Otago, Christchurch, and New Zealand Brain Research Institute, Christchurch, New Zealand

Corresponding Author: Prof. Wassilios Meissner, CRMR AMS, Hôpital Pellegrin, CHU Bordeaux, Place Amélie Raba Léon, 33076 BORDEAUX CEDEX, Phone: +33557821253, Fax: +33547304391, wassilios.meissner@chu-bordeaux.fr

The authors have no conflict of interest regarding the content of the manuscript.

Keywords: MSA, ataxia, DAT-SPECT 


\section{Abstract}

Introduction: The added value of dopamine transporter SPECT (DAT-SPECT) for the diagnosis of "possible" multiple system atrophy of the cerebellar type (MSA-C) remains unknown.

Methods: We reviewed retrospectively the charts of 128 consecutive patients with a clinical diagnosis of MSA-C who were seen between 2007 and 2016 at the French Reference Center for MSA. The main objective was to evaluate the proportion of patients for whom the diagnosis of "possible" MSA-C was made because of a positive DAT-SPECT.

Results: Seventy-eight MSA-C patients had at least one DAT-SPECT. Fifty-nine of them were considered for the final analysis. In these, 22 had "possible" MSA-C and 23 "probable" MSA-C before DAT-SPECT, while 14 did not reach diagnosis criteria at that time. In those with "possible" MSA-C, DAT-SPECT was positive in $64 \%$. In patients with "probable" MSA-C, 83\% showed nigrostriatal denervation. Six out of 14 (43\%) received a diagnosis of "possible" MSA-C because of positive DAT-SPECT. These patients had mean disease duration of 2.3 years at the time of DAT-SPECT compared to 3.5 years of the entire cohort of MSA-C patients with DAT-SPECT. Of the eight remaining, one had positive DAT-SPECT but also pons atrophy on magnetic resonance imaging, and seven progressed to "probable" MSA based on clinical features.

Conclusion: Our results suggest that DAT-SPECT significantly contributes to the diagnosis of "possible" MSA-C (43\% of patients not reaching consensus diagnosis criteria before DAT-SPECT). DAT-SPECT seems especially useful in patients with shorter disease duration, while a negative result does not exclude a diagnosis of MSA. 


\section{Introduction}

Multiple system atrophy (MSA) is a rare and fatal neurodegenerative disorder characterized by a variable combination of parkinsonism, cerebellar ataxia and autonomic dysfunction. Consensus diagnosis criteria distinguish two clinical subtypes of MSA, the parkinsonian variant with predominant parkinsonism (MSA-P) and the cerebellar variant with prominent cerebellar features (MSA-C) [1, 2]. Three levels of certainty were established, i.e. "possible", "probable" and "definite" MSA, the latter requiring post-mortem confirmation. An early diagnosis is important for the clinical guidance of patients and caregivers because of the severe prognosis of MSA-C compared to other conditions, in particular sporadic adult-onset ataxia (SAOA) of unknown etiology [3]. To enhance the diagnostic sensitivity in early disease, the revision of the consensus criteria in 2008 has included additional features such as presynaptic nigrostriatal dopaminergic denervation on SPECT or PET for the diagnosis of "possible" MSA-C. According to these criteria, the diagnosis of "possible" MSA-C requires the presence of cerebellar impairment, plus at least one feature suggesting autonomic dysfunction (i.e. otherwise unexplained urinary urgency, frequency or incomplete bladder emptying, erectile dysfunction in males, or significant orthostatic blood pressure decline that does not meet the level required in "probable" MSA) and at least one additional feature (e.g. presynaptic nigrostriatal denervation on dopamine transporter (DAT)-SPECT). Hitherto, the added diagnostic value of dopamine transporter DAT-SPECT for the diagnosis of "possible" MSA-C remains unknown.

We conducted a retrospective study at the two sites of the French Reference Center for MSA to assess the contribution of DAT-SPECT to the diagnosis of 
"possible" MSA-C in patients who eventually received a clinical diagnosis of MSA, but who did not fulfill consensus diagnosis criteria at the time of DAT-SPECT.

\section{Methods}

\section{Patient selection}

We reviewed the charts of all consecutive MSA-C patients of the longitudinal cohort of 414 MSA patients (MSA-C $=128, M S A-P=286)$ who were recruited at the French Reference Centre for MSA in Bordeaux or Toulouse between 2007 and June 2016. The clinical diagnosis of MSA was made following current consensus criteria [2]. These criteria were also applied in patients who were diagnosed before 2008. Postmortem confirmation was obtained in 3 MSA-C patients who donated their brain after death.

For the purpose of this study, we referred to the data that were collected at the visit prior or at the time of DAT-SPECT, including main clinical and imaging features of the second consensus statement: clinical signs of cerebellar impairment (gait ataxia, limb ataxia, dysarthria, oculomotor dysfunction), parkinsonism (bradykinesia, rigidity, tremor, postural instability), autonomic dysfunction (orthostatic hypotension and urinary dysfunction), pyramidal signs, stridor and features of MSA-C on brain magnetic resonance imaging (MRI; atrophy of putamen, middle cerebellar peduncles, or pons). MRI was available for all but one patient. MRI was performed before in 36, at the same time in 30, and after DAT-SPECT in two patients.

In patients without DAT-SPECT, data of the first visit at the French Reference Center for MSA were used for the analysis. All patients gave written informed consent to personal data processing for research purposes and all necessary regulatory approvals were obtained (CNIL 1338780 v1). 


\section{DAT-SPECT}

The main objective was to evaluate the proportion of patients for whom the diagnosis of "possible" MSA-C was made because of a positive DAT-SPECT, as the only additional feature of current consensus diagnosis criteria [2]. A specialist in nuclear medicine $(\mathrm{FH})$, who was blinded with regard to the clinical diagnosis, performed a visual analysis as well as a semi-quantitative assessment of all exams with the DaTsoft3D software (Toulouse, France, http://www.en.datsoft3d.com). According to current recommendations [4], the visual analysis was used as the primary criterion and the semi-quantitative assessment as the secondary criterion. DaTsoft3D software quantification provides an estimation of spatial resolution (FWHM) directly on striatal images before quantification and an automatic registration of a 3D MRIbased template of caudate and putamen (right and left). Threshold was set at 2.2 based on the results of the PPMI international multicenter database (http://www.ppmi-info.org/). For the sake of standardization and since all images were analyzed with the same software, the assessment was limited to patients with DAT-SPECT performed at the two sites of the French Reference Center for MSA (59 out of 78 , figure 1$)$.

\section{Statistical analysis}

Chi-square or Fisher tests were used for the comparison of categorical data and a Mann-Whitney $U$ test for the comparison of age at onset and disease duration between patients with and without DAT-SPECT (supplementary table 1). For the data analysis of the three patient groups with DAT-SPECT, chi-squared tests were used for analyzing categorical data, a Mann-Whitney $U$ test for comparing disease duration 
between patients with positive and negative DAT-SPECT within groups, and a oneway ANOVA for comparing age at onset and disease duration between the three groups (supplementary tables 2 and 3). A one-way ANOVA was used to compare DAT-SPECT binding potentials (BP) between groups and a Pearson correlation analysis was performed between BP and disease duration. Data are presented as mean \pm standard deviation or proportion. A $p<0.05$ was considered significant. All tests were performed by using Prism 7.

\section{Results}

Demographic, clinical and MRI data

Of the entire population of patients with a diagnosis of MSA-C at final follow-up, $53.1 \%$ were men, the mean age at disease onset was $58.9 \pm 7.7$ years and the mean disease duration at the first visit was $3.8 \pm 3.0$ years. Bradykinesia, cerebellar oculomotor dysfunction, severe orthostatic hypotension and urinary dysfunction were more frequent in patients without DAT-SPECT (supplementary table 1). These patients also showed longer disease duration at the first visit, but none of these differences reached significance after Bonferroni correction for multiple comparisons.

Of the 59 MSA-C patients with DAT-SPECT considered for the analysis (figure 2), "possible" MSA-C was diagnosed in 22 and "probable" MSA in 23 before DATSPECT. According to current consensus criteria, evidence for a diagnosis of MSA-C was insufficient in 14 patients before DAT-SPECT. An overall comparison between these three groups showed significant differences in disease duration at DATSPECT, as well as in the proportion of limb ataxia, parkinsonism, bradykinesia, rigidity, tremor and autonomic dysfunction (supplementary table 2). A higher proportion of patients with "possible" and "probable" MSA-C showed classical MRI 
features of MSA compared to subjects with insufficient criteria at the time of DATSPECT. However, group differences were not statistically significant except for a strong trend for putamen atrophy (supplementary table 3).

\section{DAT-SPECT}

In patients with "possible" MSA-C, DAT-SPECT was positive in 14 (64\%). Mean disease duration at DAT-SPECT was $4.8 \pm 3.3$ years for those with positive and $3 \pm$ 1.7 years for those with negative DAT-SPECT (not significant). In patients with "probable" MSA-C, 19 (83\%) showed nigrostriatal denervation. Mean disease duration at DAT-SPECT was $4.1 \pm 2.5$ years for those with positive and $2.3 \pm 1.9$ years for those with negative DAT-SPECT (trend for longer disease duration in "probable" MSA-C patients with positive DAT-SPECT, $p=0.063$ ).

In subjects with insufficient criteria at the time of DAT-SPECT, four had isolated ataxia, five had ataxia with features suggesting autonomic dysfunction and five had ataxia with one additional feature. Of these 14 patients, six had a positive DAT-SPECT with three qualifying immediately after DAT-SPECT for a diagnosis of "possible" MSA-C according to revised consensus criteria. In the remaining three, the diagnosis of "possible" MSA-C was only reached 10-18 months later because of the onset of features suggesting autonomic dysfunction. These six patients had a mean disease duration of $2.3 \pm 0.8$ years at DAT-SPECT and progressed to "probable" MSA during follow-up. DAT-SPECT was positive in another patient but there was also pons atrophy on MRI as additional feature. In other terms, the positive DATSPECT did not provide significant additional information for the diagnosis. One year later, this patient developed features suggesting autonomic dysfunction and parkinsonism qualifying for the diagnosis of "possible" MSA-C. Seven patients did not 
show significant denervation. These patients reached consensus criteria 4.6 years after symptom onset and had a diagnosis of "probable" MSA at last follow-up. They tended to be younger (57.7 vs 64.9 years at disease onset), while disease duration and clinical presentation at the first visit were not different compared to patients with insufficient criteria and positive DAT-SPECT.

A second DAT-SPECT (interval between the first and second scan $=2.6 \pm 2.6$ years) was performed in five of the seven subjects with initial negative result and insufficient evidence for a diagnosis of "possible" MSA-C. Among these, DAT-SPECT became positive in two. However, at the time of the second DAT-SPECT, the diagnosis was already "probable" MSA-C because of severe autonomic failure (urinary incontinence in one patient and severe orthostatic hypotension in the other). A third DAT-SPECT (interval between the second and third scan $=8$ and 17 months) was performed in two patients, yielding a positive result in one subject. Again, the clinical diagnosis had already progressed to "probable" MSA-C because of urinary incontinence.

Overall, the proportion of patients with a positive DAT-SPECT was significantly different between the three groups at the time of imaging (insufficient evidence (43\%), "possible" (64\%) and "probable" MSA (83\%), chi $\left.^{2}=6.2, p<0.05\right)$. Of all patients with a positive DAT-SPECT, $21 / 40(52.5 \%)$ had no bradykinesia and $24 / 40(60 \%)$ no parkinsonism on clinical examination.

\section{Quantitative assessment of DAT-SPECT}

The mean BP in the putamen were $2.1 \pm 1.0$ for patients with "probable" MSA-C (1.7 \pm 0.7 in patients with positive DAT-SPECT vs. $3.8 \pm 0.7$ in patients with negative DAT-SPECT), $2.6 \pm 1.1$ for patients with "possible" MSA-C (2.1 \pm 0.7 vs. $3.6 \pm 0.8)$ 
and $2.7 \pm 1.2$ for patients not reaching diagnosis criteria for MSA-C ( $2.0 \pm 0.8$ vs. 3.7 \pm 0.9 ) at the time of DAT-SPECT. Differences in mean BP between groups were not

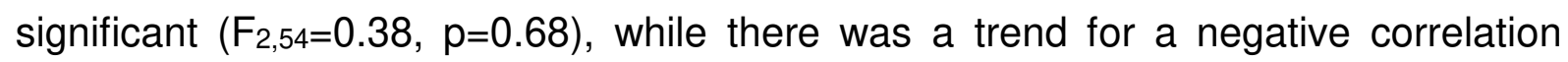
between BP and disease duration ( $r=-0.23, p=0.086)$.

The mean BP in the caudate were $3.7 \pm 1.3$ for patients with "probable" MSAC (3.4 \pm 1.2 vs. $5.2 \pm 1.1), 3.6 \pm 0.9$ for patients with "possible" MSA-C (3.2 \pm 0.8 vs. $4.2 \pm 0.9)$ and $4.0 \pm 1.3$ for patients not reaching the criteria for MSA-C (3.5 \pm 1.3 vs. $4.7 \pm 0.9)$ at the time of DAT-SPECT. Differences in mean BP between groups were

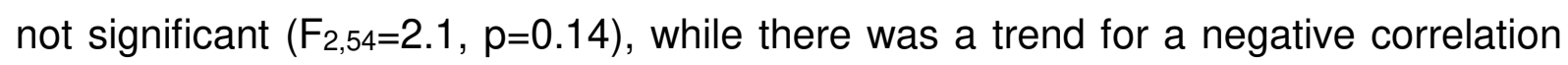
between BP and disease duration $(r=-0.23, p=0.086)$.

The mean BP in the striatum were $2.5 \pm 1.0$ for patients with "probable" MSAC ( $2.2 \pm 0.7$ vs. $3.9 \pm 0.8), 2.7 \pm 0.9$ for patients with "possible" MSA-C (2.3 \pm 0.6 vs. $3.5 \pm 0.7)$ and $2.9 \pm 1.1$ for patients not reaching the criteria for MSA-C $(2.3 \pm 0.9$ vs. $3.7 \pm 0.8)$ at the time of DAT-SPECT. Differences in mean BP between groups were not significant $\left(F_{2,54}=0.8, p=0.45\right)$, while there was a trend for a negative correlation between $\mathrm{BP}$ and disease duration $(r=-0.24, \mathrm{p}=0.07)$.

\section{Discussion}

In our retrospective study, DAT-SPECT allowed the diagnosis of "possible" MSA-C in six patients $(43 \%)$ not reaching the diagnosis criteria of MSA at the time of imaging. DAT-SPECT was therefore significantly contributive to the diagnosis of "possible" MSA-C in these patients, while the overall contribution was relatively low (6/59) when considering the entire cohort of MSA-C patients followed at the French Reference Center for MSA. One reason may be that patient referral to tertiary centers only occurs once clinical core and supportive features for "possible" and "probable" MSA 
are already present. This is corroborated by the mean disease duration of almost four years between symptom onset and first visit. Accordingly, DAT-SPECT might be more useful at earlier stages of the disease in patients seen at non-reference centers. However, the increasing proportion of striatal dopaminergic denervation in MSA-C patients with higher level of diagnostic certainty and the trend for a negative correlation between BP and disease duration suggest that the likelihood of a positive DAT-SPECT decreases with shorter disease duration. In addition, although the mean disease duration at the first visit at our center was only 2.1 years in the 14 patients with insufficient criteria at the time of DAT-SPECT, the mean disease duration at the time of their clinical MSA-C diagnosis was 3.6 years illustrating the challenges for an early diagnosis in this population.

Although no longer necessary according to consensus diagnosis criteria, DATSPECT was frequently performed at the French Reference Center for MSA in patients who had already a clinical diagnosis of MSA-C. This illustrates that even expert clinicians attempt to increase the diagnostic certainty for a devastating neurodegenerative disease such as MSA beyond established diagnosis criteria. In the present study, $73.3 \%$ of patients with a diagnosis of "possible" or "probable" MSA-C at the time of imaging had a positive DAT-SPECT. This number is similar compared to the $76.9 \%$ reported by a smaller study including 13 MSA-C patients [5]. The increasing proportion with higher diagnostic certainty and the trend for a negative correlation with disease duration further suggest that striatal dopaminergic denervation occurs progressively in MSA-C, as also illustrated by the progressive appearance of parkinsonism and the moderate to severe neuronal loss in the substantia nigra at the time of death [6]. It should be emphasized that a negative DAT-SPECT does not exclude the diagnosis of MSA since this was observed in 
almost one third of patients with a clinical diagnosis of MSA-C. Patients with shorter disease duration were more likely to have a negative DAT-SPECT, as was also observed in a small series of six MSA-C patients with a mean disease duration of 1.6 years [7]

Not surprisingly, more MSA-C patients without DAT-SPECT had received their diagnosis before 2008, the year the revised consensus diagnosis criteria were published. Additionally, the trend for more bradykinesia, urinary dysfunction, severe orthostatic hypotension, and longer disease duration at the first visit made it also less likely to perform DAT-SPECT in these patients.

As illustrated by the high number of MSA-C patients in our study without parkinsonian signs despite striatal dopaminergic denervation, DAT-SPECT is able to detect subclinical abnormalities, thereby increasing the diagnostic certainty for MSAC compared to SAOA, one major differential diagnosis. In contrast, patients with Fragile X-associated tremor/ataxia syndrome (FXTAS) or spinocerebellar ataxia (SCA), two additional differential diagnoses of MSA-C, may also show dopaminergic denervation $[8,9]$. Of note, middle cerebellar peduncle hyperintensities on brain MRI can be found in MSA-C and FXTAS, usually with a distinct topography and additional white matter lesions in FXTAS [9]. The "hot cross bun sign", witnessing a loss of myelinated transverse pontocerebellar fibers, may also be found in SCA 2, but patients are usually younger and have a family history of autosomal dominant ataxia $[10]$

The main limitation is the retrospective nature of the study. In addition, although we considered a large cohort of 128 patients with a diagnosis of MSA-C at last follow-up, the main result relies on only 14 patients with insufficient criteria for "possible" MSA-C at the time of DAT-SCAN. This low overall contribution of DAT- 
SPECT may be due to the patient referral to tertiary centers only in more advanced disease, which is corroborated by the long mean interval between symptom onset and first visit.

In conclusion, our results suggest that DAT-SPECT contributes significantly to the diagnosis of "possible" MSA-C. DAT-SPECT seems especially useful in patients with shorter disease duration, while a negative result does not exclude a diagnosis of MSA. Additional studies are warranted to confirm our main result in larger cohorts.

Author roles: (1) conception and design of the study, or acquisition of data, or analysis and interpretation of data, (2) drafting the article or revising it critically for important intellectual content, (3) final approval of the version to be submitted.

Sylvain Vergnet, Alexandra Foubert-Samier, Anne Pavy-Le Traon, Wassilios G. Meissner: (1) (2) (3). Florent Hives, Julia Dupouy, Christine Brefel-Courbon, Fabienne Ory-Magne: (1) (3). Pierre Payoux, Philippe Fernandez, Marie Meyer, Olivier Rascol, François Tison: (2) (3).

\section{Author Disclosures (past 12 months)}

Sylvain Vergnet, Florent Hives, Alexandra Foubert-Samier, Pierre Payoux, Philippe Fernandez have nothing to disclose.

Julia Dupouy has received travel grants from Teva, UCB, Bastide and Orkyn.

Christine Brefel-Courbon has received grants from $\mathrm{CHU}$ de Toulouse, FranceParkinson, Programme Hospitalier de Recherche Clinique, and has received fees for serving as advisory board member for Zambon, for consultancy activities from Teva, UCB, Aguettant, Abbvie, Orkyn. 
Fabienne Ory-Magne has received grants from Orkyn, Medtronic, Abbvie, Aguettant; travel grants from Orkyn, Medtronic, Abbvie and teaching honoraria from UCB, Aguettant, Orkyn, Abbvie, Zambon.

Olivier Rascol has received grants from Agence Nationale de la Recherche (ANR), CHU de Toulouse, France-Parkinson, INSERM-DHOS Recherche Clinique Translationnelle, MJ Fox Foundation, Programme Hospitalier de Recherche Clinique, European Commission (FP7, H2020) and has served as scientific consultant for AbbVie, Adamas, Acorda, Addex, AlzProtect, Apopharma, Astra Zeneca, Bial, Biogen, Britannia, Clevexel, Cynapsus, INC Reasearch, Lundbeck, Merck, MundiPharma, Neuroderm, Novartis, Oxford Biomedica, Parexel, Pfizer, Prexton Therapeutics, Quintiles, Sanofi, Servier, Teva, UCB, XenoPort, Zambon.

François Tison received a travel grant from ORKYN, and research grants from French Health Ministry (PHRC), outside the presented work.

Anne Pavy-Le Traon has received research grants from the French Health Ministry, and the European Community.

Wassilios G. Meissner has received fees for editorial activities with Springer, for consultancy activities from Affiris, Biohaven, Lundbeck and Sanofi, teaching honoraria from UCB and MDS, as well as research support from the Michael J Fox Foundation, the University Hospital Bordeaux, the French Health Ministry, the European Community, ANR, ARAMISE, PSP-France, MSA Coalition, ARAMISE, LABEX Excellence Initiative. 


\section{References}

[1] Gilman S, Low PA, Quinn N, Albanese A, Ben-Shlomo Y, Fowler CJ, et al. Consensus statement on the diagnosis of multiple system atrophy. J Neurol Sci. 163 (1999) 94-8.

[2] Gilman S, Wenning GK, Low PA, Brooks DJ, Mathias CJ, Trojanowski JQ, et al. Second consensus statement on the diagnosis of multiple system atrophy. Neurology. 71 (2008) 670-6.

[3] Giordano I, Harmuth F, Jacobi H, Paap B, Vielhaber S, Machts J, et al. Clinical and genetic characteristics of sporadic adult-onset degenerative ataxia. Neurology. 89 (2017) 1043-9.

[4] Darcourt J, Booij J, Tatsch K, Varrone A, Vander Borght T, Kapucu OL, et al. EANM procedure guidelines for brain neurotransmission SPECT using (123)Ilabelled dopamine transporter ligands, version 2. Eur J Nucl Med Mol Imaging. 37 (2010) 443-50.

[5] Munoz E, Iranzo A, Rauek S, Lomena F, Gallego J, Ros D, et al. Subclinical nigrostriatal dopaminergic denervation in the cerebellar subtype of multiple system atrophy (MSA-C). J Neurol. 258 (2011) 2248-53.

[6] Ozawa T, Paviour D, Quinn NP, Josephs KA, Sangha H, Kilford L, et al. The spectrum of pathological involvement of the striatonigral and olivopontocerebellar systems in multiple system atrophy: clinicopathological correlations. Brain. 127 (2004) 2657-71. 
[7] Nicastro N, Garibotto V, Burkhard PR. 123I-FP-CIT SPECT Accurately distinguishes parkinsonian from cerebellar variant of multiple system atrophy. Clin Nucl Med. 43 (2018) e33-e6.

[8] Apartis E, Blancher A, Meissner WG, Guyant-Marechal L, Maltete D, De Broucker T, et al. FXTAS: new insights and the need for revised diagnostic criteria. Neurology. 79 (2012) 1898-907.

[9] Renaud M, Perriard J, Coudray S, Sevin-Allouet M, Marcel C, Meissner WG, et al. Relevance of corpus callosum splenium versus middle cerebellar peduncle hyperintensity for FXTAS diagnosis in clinical practice. J Neurol. 262 (2015) $435-42$.

[10] Lee YC, Liu CS, Wu HM, Wang PS, Chang MH, Soong BW. The 'hot cross bun' sign in the patients with spinocerebellar ataxia. Eur J Neurol. 16 (2009) 513-6. 


\section{Figure captions}

Figure 1: Study profile

Figure 2: Diagnostic certitude before performing DAT-SPECT 


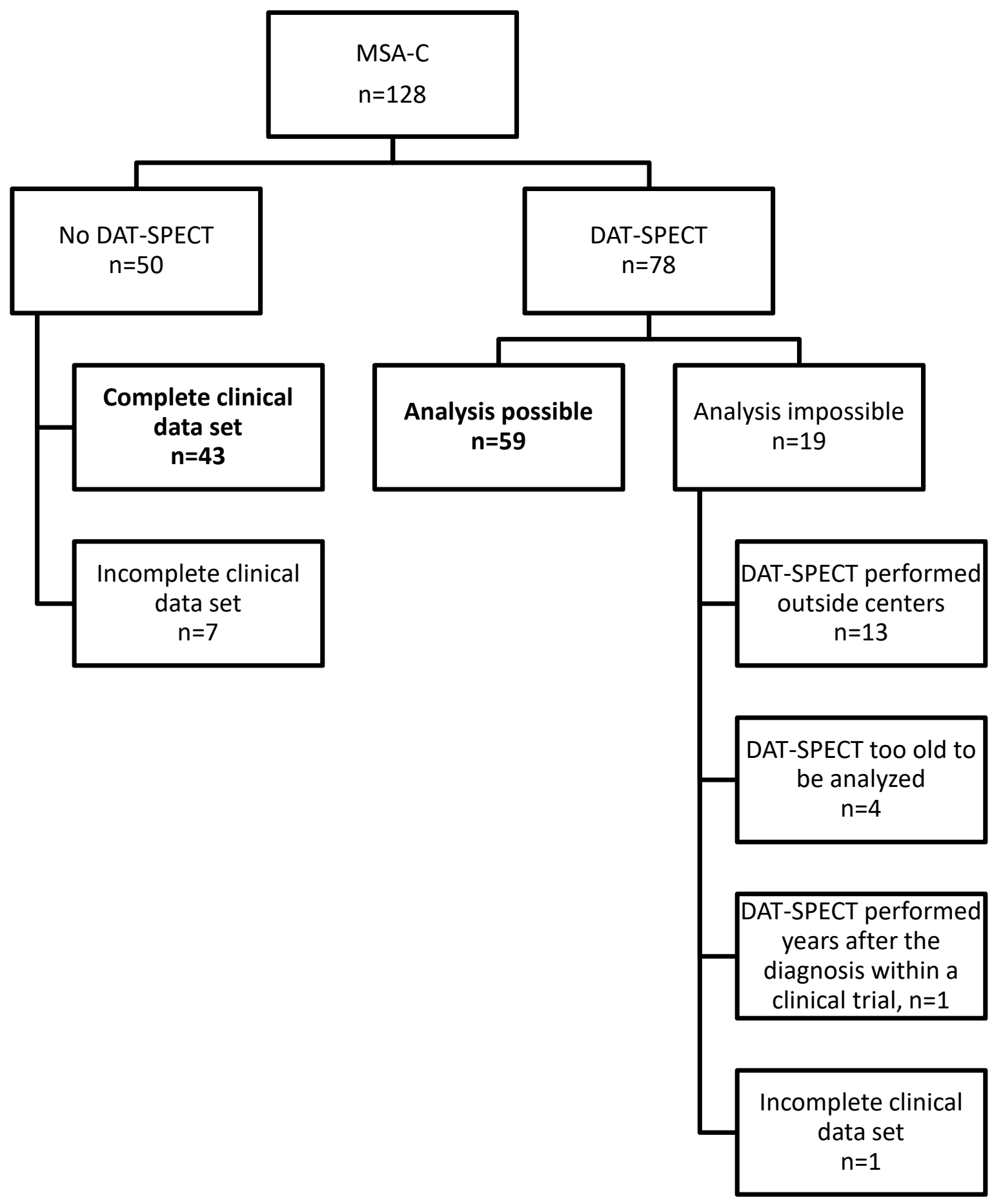

Figure $1 \quad$ Study profile 


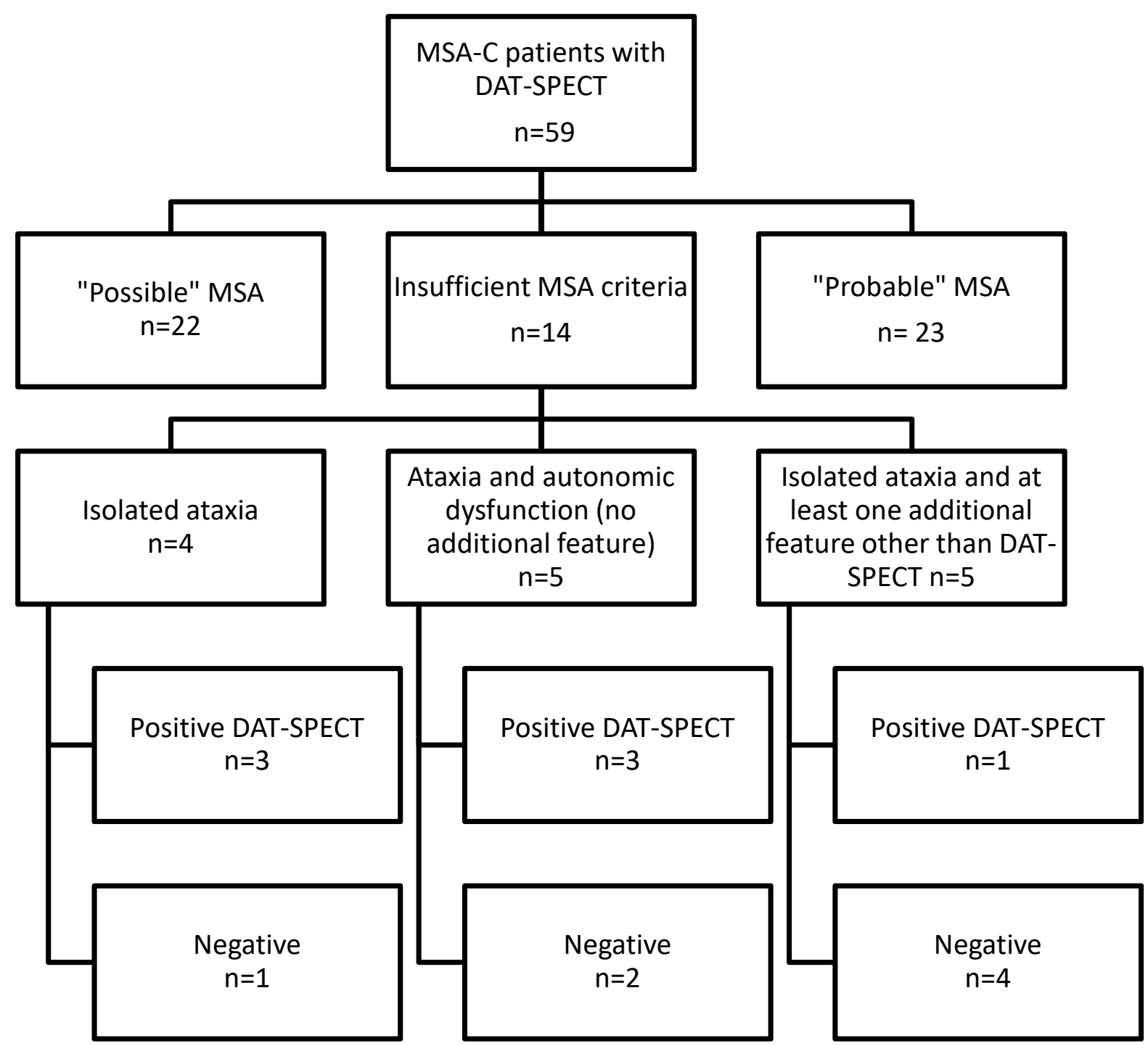

Figure 2 Diagnostic certitude before performing DAT-SPECT 